\title{
Doença renal crônica em adultos de Rio Branco, Acre: inquérito de base populacional
}

\author{
Chronic kidney disease among adults in Rio Branco, State of Acre: a \\ population-based survey
}

Thatiana Lameira Maciel Amaral (https://orcid.org/0000-0002-9197-5633) ${ }^{1}$

Cledir de Araújo Amaral (https://orcid.org/0000-0002-7221-5364) ${ }^{2}$

Maurício Teixeira Leite de Vasconcellos (https://orcid.org/0000-0003-1658-2589) ${ }^{3}$

Gina Torres Rego Monteiro (https://orcid.org/0000-0002-9900-1825) ${ }^{4}$

${ }^{1}$ Programa de PósGraduação em Saúde Coletiva, Centro de Ciências da Saúde e do Desporto, Universidade Federal do Acre. BR $364 \mathrm{Km} 04$ Distrito Industrial, Campus Universitário. 69920-900 Rio Branco AC Brasil. thatianalameira27@ gmail.com

${ }^{2}$ Instituto Federal de Educação, Ciência e Tecnologia do Acre. Rio Branco AC Brasil.

${ }^{3}$ Escola Nacional de Ciências Estatísticas, Instituto Brasileiro de Geografia e Estatística. Rio de Janeiro RJ Brasil.

${ }^{4}$ Escola Nacional de Saúde Pública Sérgio Arouca, Fundação Oswaldo Cruz. Rio de Janeiro RJ Brasil.

\begin{abstract}
The scope of this study was to establish the prevalence of CKD and associated factors among young adults (18-59 years of age) from Rio Branco in the State of Acre. It involved a population-based survey conducted in 2014, in the urban and rural areas of the municipality and CKD was defined as the glomerular filtration rate $(G F R)<$ $60 \mathrm{ml} / \mathrm{min} / 1.73 \mathrm{~m}^{2}$, estimated by the CKD-EPI, and the presence of albuminuria $>29 \mathrm{mg} / \mathrm{g}$. Association measures were estimated by logistic regression, with a confidence level of $95 \%$. The overall prevalence of CKD was $6.2 \%$. The presence of CKD was higher among women, aged 40 to 59 years, with non-white skin color, with lower schooling, and of sedentary disposition. There were statistically significant differences in the distribution according to the presence of CKD in the hypertension, diabetes and hospitalization variables over the past 12 months. CKD among adults was associated with the female sex (OR: 2.41, 95\%CI: 1.14-5.12), diabetes (OR: 4.67, 95\%CI: 1.28-17.03) and arterial hypertension (OR: 1.98; 95\%CI: 1.16-3.37). CKD reveals a high prevalence and is associated with chronic diseases, calling for the need for public health measures for early detection and prevention of its progression.

Key words Chronic Kidney Disease, Survey, Prevalence, Adult, Association
\end{abstract}

Resumo O objetivo do presente estudo foi verificar a prevalência de DRC e os fatores a ela associados em adultos jovens (18-59 anos) de Rio Branco, Acre. Trata-se de um inquérito de base populacional realizado em 2014, nas zonas urbana e rural do município de Rio Branco, Acre. A DRC foi definida pela taxa de filtração glomerular (TFG) $<60 \mathrm{ml} / \mathrm{min} / 1,73 \mathrm{~m}^{2}$, estimada pela CKD -EPI, e presença de albuminúria $>29 \mathrm{mg} / \mathrm{g}$. Medidas de associação foram estimadas por regressão logística, com grau de confiança de 95\%. A prevalência geral de DRC foi de 6,2\%. A presença de DRC foi maior entre as mulheres, naqueles com 40 a 59 anos, de cor da pele não-branca, com menor escolaridade e entre os sedentários. Houve diferenças estatisticamente significativas na distribuição segundo a presença de DRC nas variáveis hipertensão arterial, diabetes e internação nos últimos 12 meses. A DRC entre os adultos esteve associada ao sexo feminino (OR: 2,41; IC95\%: 1,14-5,12), diabetes (OR: 4,67; IC95\%: 1,28-17,03) e hipertensão arterial (OR: 1,98; IC95\%: 1,16-3,37). A DRC apresenta alta prevalência e está associada às doenças crônicas, evidenciando a necessidade de medidas em saúde pública para detecção precoce e prevenção da sua progressão.

Palavras-chave Doença Renal Crônica, Inquérito, Prevalência, Adulto, Associação 


\section{Introdução}

A Doença Renal Crônica (DRC) é um importante problema de saúde pública mundial que vem sofrendo influência da transição epidemiológica, resultando em altas prevalências que culminaram por despertar a atenção da comunidade científica internacional ${ }^{1}$.

O aumento da expectativa de vida e no estilo de vida da população, notadamente o sedentarismo e o excesso de peso, levam ao aumento das doenças crônicas, apontadas como fatores associados ao aumento da lesão renal em todo o mundo. O reflexo dessas alterações repercutiu no aumento da incidência de DRC na América Latina de 33,3 por milhão de habitantes em 1993, para 167,8 por milhão de habitantes em $2005^{2}$.

As doenças crônicas e seus fatores de risco, entre eles as comorbidades, podem causar graves transtornos à qualidade de vida dos indivíduos, aumentar os gastos do país com saúde, além de favorecer à ocorrência de morte prematura ${ }^{3}$. Em estudo de metanálise utilizando dados de 28 coortes para avaliar os fatores de risco para progressão da DRC, ocorrência de eventos cardiovasculares e morte, apontou que a idade esteve negativamente associados ao risco de necessidade de terapia renal substitutiva (TRS), estando a cor da pele negra, sexo masculino, hipertensão arterial e diabetes positivamente associado ao maior risco de progressão da DRC, não havendo associação com tabagismo ou história de outras doenças cardiovasculares, no entanto, estes fatores não se comportam de forma igual nos demais desfechos avaliados ${ }^{4}$.

A Política Nacional de Atenção ao Portador de Doença Renal ${ }^{5}$ prioriza a detecção precoce da DRC, bem como o tratamento adequado das suas formas leves. A descoberta da DRC nos estágios iniciais e o encaminhamento precoce para acompanhamento e tratamento são fundamentais para evitar ou retardar a progressão da doença, bem como para a diminuição de indivíduos em TRS $^{6}$.

Estratégias para prevenção, detecção, avaliação e tratamento da DRC são fundamentais para o controle dessa morbidade. Independentemente das diferenças regionais em relação aos fatores de risco e recursos para o cuidado, aumentar a eficiência na utilização de conhecimentos e recursos disponíveis favorecerá a prestação dos serviços de assistência e os resultados da DRC ${ }^{7}$. Assim, o presente estudo objetiva estimar a prevalência de DRC e os fatores a ela associados em adultos (18 a 59 anos) usando parte dos dados do Estudo de Doenças Crônicas (EDOC) em Rio Branco, Acre.

\section{Métodos}

O EDOC é composto por duas pesquisas domiciliares: EDOC-A sobre adultos (18 a 59 anos) e EDOC-I sobre idosos (60 anos ou mais), residentes em Rio Branco, Acre. Foram excluídos da população de pesquisa as mulheres grávidas e os indivíduos com comprometimentos cognitivos que inviabilizassem a comunicação ou o entendimento das perguntas. Os planos de amostragem foram selecionados em dois estágios, setor e domicílio, sendo o primeiro estágio comum às duas pesquisas. A seleção dos setores foi feita com probabilidade proporcional ao seu número e domicílios particulares no Censo Demográfico 2010 (CD2010), do IBGE. Os domicílios foram selecionados por amostragem sistemática com inícios aleatórios e intervalos distintos por pesquisa. Nos domicílios selecionados para EDOC-A todos os adultos moradores foram entrevistados.

O tamanho da amostra foi calculado considerando a prevalêncis de alteração da função renal de $15 \%$ em adultos ${ }^{7}$, com grau de confiança de 95\% e erro absoluto de $3 \%{ }^{8}$ para amostragem aleatória simples de proporções. Considerando que o plano de amostragem é conglomerado por setor, foi arbitrado um efeito de plano de amostragem de 1,95 para determinar o tamanho da amostra, que recebeu acréscimo de $20 \%$ para compensar as não-respostas esperadas. Este procedimento resultou em uma amostra de 652 adultos. Dividindo esse tamanho de amostra pelo número médio de adultos por domicílio, obtido no CD2010 e definindo a seleção de 11 domicílios por setor, foi obtido um tamanho para a amostra de setores de 40. A amostra efetiva foi de 685 adultos $^{9}$.

Os pesos amostrais foram calculados pelo inverso das probabilidades de inclusão em cada estágio e foram posteriormente calibrados para dados populacionais por sexo e grupos de idade, usando um estimador de pós-estratificação de forma a lidar com os vieses típicos das pesquisas domiciliares e corrigir não-respostas diferenciais ${ }^{10}$. Os dados populacionais usados na calibração dos pesos amostrais foram estimados para $1^{\circ}$ de julho de 2014, usando o método da tendência linear ${ }^{11}$ que o IBGE aplica em suas estimativas populacionais por município. No presente estudo foram incluídos todos os adultos que realizaram avaliação laboratorial da creatinina sérica, ou seja, 649 adultos (451 mulheres e 198 homens), que correspondem a uma subamostra de informações completas sobre o tema. Os pesos amostrais dessa subamostra foram 
corrigidos e novamente calibrados para produzir estimativas para 211.902 adultos (110.278 mulheres e 101.624 homens). Maiores detalhes sobre o plano de amostragem do EDOC, cálculo e calibração dos pesos da amostra e subamostras ver artigo de Amaral et al. ${ }^{12}$.

A amostra final da presente pesquisa foi de 649 adultos, considerando os pesos amostrais decorrente do delineamento de amostras complexas, permitiu a expansão para 211.902 indivíduos, onde 110.503 eram mulheres $(52,1 \%)$ e 101.399 (47,9\%) homens.

Os procedimentos para a coleta de dados foram realizados por pessoal treinado e supervisionados pela equipe de coordenação, sendo refeitas cerca de $10 \%$ das entrevistas e avaliações físicas para controle de qualidade das informações. Deste modo, o trabalho de campo compreendeu quatro etapas, a saber: atualização do cadastro de domicílios dos setores censitários sorteados e seleção dos domicílios, entrevista das pessoas elegíveis, avaliação física e coleta de amostras biológicas, e retorno dos resultados dos exames laboratoriais aos participantes.

$\mathrm{Na}$ avaliação física foram coletados dados antropométricos que incluíram a medição do peso, altura e circunferência da cintura, quadril, braço e panturrilha, seguindo os protocolos preconizados pelo American College of Sports Medicine $(\mathrm{ACSM})^{13}$, e também foram verificados os sinais vitais por meio da mensuração da pressão arterial, frequência respiratória e cardíaca, todos em duplicata, sendo consideradas as médias das aferições.

$\mathrm{O}$ peso foi mensurado por meio de uma balança digital Bal Gl 200 da G-Tech ${ }^{\circledR}$ com resolução de 50 gramas disposta em superfície plana. Os participantes foram orientados e estavam usando roupas leves e convidados a subir descalços e com os bolsos vazios no centro da base da balança, com o corpo ereto e peso distribuído uniformemente entre os dois pés, braços ao lado do corpo e olhando para frente.

A altura dos participantes foi determinada por um estadiômetro portátil Sanny ${ }^{\circledR}$, com resolução em milímetros e a base disposta sempre sobre uma superfície plana. O participante, sem utilizar objetos na cabeça, como bonés ou enfeites de cabelo, disposto de costas para o antropômetro, com pernas e pés paralelos, peso distribuído em ambos, braços lateralizados e palmas das mãos voltadas para o corpo. Após o alinhamento da parte de trás da cabeça, costas, nádegas, pernas e calcanhares e olhar para frente utilizando o plano de Frankfurt para o posicionamento da cabeça, era solicitado ao indivíduo inspirar profundamente e prender a respiração durante a aferição, realizada deslocando a parte móvel do estadiômetro até o ponto mais alto da cabeça comprimindo os cabelos o suficiente para obter a mensuração da altura.

A determinação do índice de massa corporal (IMC) resultou do cálculo da razão do peso $(\mathrm{kg})$ pela altura (em metros) ao quadrado $\left(\mathrm{m}^{2}\right)$. Para análise, foi classificado em baixo peso $<18,5 \mathrm{~kg} /$ $\mathrm{m}^{2}$; peso normal de 18,5 a $24,9 \mathrm{~kg} / \mathrm{m}^{2}$; sobrepeso 25 a $29,9 \mathrm{~kg} / \mathrm{m}^{2}$; e obeso $\geq 30 \mathrm{~kg} / \mathrm{m}^{214}$.

A pressão arterial (PA) foi mensurada de acordo com o protocolo recomendado pela Sociedade Brasileira de Cardiologia que preconiza a aferição 30 minutos ou mais após a última ingestão de cafeína ou de cigarro fumado, sendo feitas três medições: uma após 5 minutos de repouso inicial e outras duas em intervalos de 2 minu$\operatorname{tos}^{15}$. Para a aferição da PA, foi utilizado o aparelho digital da marca Beurer ${ }^{\circledR}$. O valor final da PA foi calculado pela média aritmética das segunda $\mathrm{e}$ terceira medidas. Foram, ainda, verificadas a frequência cardíaca e respiratória na última aferição da PA, bem como, a avaliação da pulsação.

Todas as análises de material biológico foram realizadas no mesmo laboratório para garantir a padronização dos métodos. Os resultados foram agrupados em formulário próprio. As amostras de sangue foram obtidas por meio da coleta de sangue periférico, com antissepsia prévia da fossa antecubital dos participantes. Parte da amostra colhida, $04 \mathrm{ml}$, foi acondicionada em tubo de ensaio a vácuo sem anticoagulante e centrifugada a $1.500 \mathrm{rpm}$ por 15 minutos. O soro extraído foi acondicionado para dosagem bioquímica de triglicérides, colesterol total e frações (HDL - lipoproteína de alta densidade e LDL - lipoproteína de baixa densidade).

O colesterol total foi dosado pelo método enzimático colorimétrico COD/PAD, da mesma forma que as frações (HDL, LDL e VLDL) e os triglicerídeos GPO/PAP (Labtest diagnostica). O colesterol é uma molécula insolúvel que circula associado a lipoproteínas (HDL, LDL e VLDL). O LDL foi obtido do VLDL por hidrólise de diferentes enzimas lipolíticas. A creatinina sérica foi dosada pelo método enzimático rastreável de espectrometria de massa de diluição com isótopos IDMS em um analisador automático (Labmax 240 Premium).

Para a análise da glicemia sérica foi utilizada amostra de $04 \mathrm{ml}$ de sangue acondicionada em tubo a vácuo contendo $2 \mathrm{mg} / \mathrm{ml}$ de fluoreto de sódio centrifugados antes das análises. A glicemia 
sérica foi dosada por meio método glicose oxidase (Labtest Diagnóstica).

Para a amostra de urina, foram coletados aproximadamente $50 \mathrm{ml}$ do jato médio da primeira urina da manhã de cada indivíduo. As amostras acondicionadas em frascos padrão e transportadas do local de coleta até o laboratório para análise em temperatura controlada.

Para o exame de urina tipo I (EAS) foi realizada análise física, química e microscópica. A creatinina urinária foi dosada pelo método enzimático de Trinder (Kit Creatinina Enzimática, Labtest) e a albuminúria pelo método de imunoturbidimetria (Kit Turbiquest Plus, Labtest). A albuminúria é definida como uma razão de albumina para creatinina de $30 \mathrm{mg} / \mathrm{g}$ ou mais, sendo considerado o valor de 30 a 299 de A2 ou moderadamente aumentado e $\geq 300 \mathrm{mg} / \mathrm{g}$ de A3 ou acentuadamente aumentado ${ }^{16}$.

A HAS foi definida como pressão arterial diastólica (PAD) $\geq 90 \mathrm{mmHg}$ e/ou pressão arterial sistólica (PAS) $\geq 140 \mathrm{mmHg}$ e/ou uso atual de medicação anti-hipertensiva ${ }^{15}$.

A presença de diabetes, foi definida de acordo com os critérios da American Diabetes Association (ADA) como sendo glicose no plasma em jejum $\geq 126 \mathrm{mg} / \mathrm{dl}$, bem como a utilização de hipoglicemiante oral ou de insulina ${ }^{17}$.

A dislipidemia foi definida pela presença de níveis anormais de um ou mais dos seguintes componentes lipídicos do sangue: colesterol total $\geq 200 \mathrm{mg} / \mathrm{dl}$; LDL-C $\geq 160 \mathrm{mg} / \mathrm{dl}$; triglicerídeos $\geq 150 \mathrm{mg} / \mathrm{dl}$; e HDL-C em homens $<40 \mathrm{mg} / \mathrm{dl}$ e mulheres $<50 \mathrm{mg} / \mathrm{dl}$, além do histórico de uso de medicamentos para redução desses valores. Para indivíduos com idade inferior a 20 anos, os pontos de corte são: colesterol total $\geq 170 \mathrm{mg} / \mathrm{dl}$, LDL-C $\geq 130 \mathrm{mg} / \mathrm{dl}$; triglicerídeos $\geq 130 \mathrm{mg} / \mathrm{dl}$ e/ ou HDL-C $\leq 45 \mathrm{mg} / \mathrm{dl}^{18}$.

Para efeito de diagnóstico de Síndrome Metabólica, os dados foram interpretados segundo a I Diretriz Brasileira de Diagnóstico e Tratamento da Síndrome Metabólica, que adotou a íntegra do estabelecido pelo National Cholesterol Education Program's Adult Treatment Panel III, cujos critérios requerem o achado de três ou mais dos seguintes componentes: i) diâmetro de cintura > $102 \mathrm{~cm}$ para homens e $>88 \mathrm{~cm}$ para mulheres; ii) triglicérides $\geq 150 \mathrm{mg} / \mathrm{dL}$; iii) HDL-colesterol $<40 \mathrm{mg} / \mathrm{dL}$ para homens e $<50 \mathrm{mg} / \mathrm{dL}$ para mulheres; iv) PAS $\geq 130 \mathrm{mmHg}$, PAD $\geq 85 \mathrm{mmHg}$, ou uso de antihipertensivo; v) glicemia de jejum $\geq 110 \mathrm{mg} / \mathrm{dL}$ ou uso de hipoglicemiante ${ }^{19}$.

Em 2012 foram definidas as categorias de risco para a progressão da doença renal crônica com base na creatinina sérica, com correção pelas fórmulas para obtenção da TFG, e da albuminúria, sendo ainda dividido o estágio 3 da DRC em $3 a$ e 3b. Destaque importante da diretriz é a adoção de categorias de risco do prognóstico de DRC, classificado de baixo a alto o risco de progressão para DRC e outras complicações ${ }^{16}$.

A variável dependente do estudo foi definida segundo os critérios da KDIGO (2012), como a presença de alteração na função renal segundo fórmula CKD-EPI com TFG $<60 \mathrm{ml} / \mathrm{min} / 1,73$ $\mathrm{m}^{2}$ e/ou com presença de albuminúria $>29 \mathrm{mg} / \mathrm{g}$ 5,14. A amostra de sangue e urina foi realizada em um único momento no tempo, sendo este método utilizado por outros estudos epidemiológicos para avaliação da prevalência e fatores associados em adultos ${ }^{20,21}$.

A amostragem complexa adotada na pesquisa implicou a necessidade de utilizar a estimação da variância entre as médias obtidas por unidades primárias de amostragem dentro de cada estágio. A análise dos dados foi feita utilizando as rotinas do Complex samples do programa Statistical Package for the Social Sciences (SPSS), versão 20.0, para Windows. Foram mantidos nos arquivos de dados o peso natural do desenho, o estrato de seleção da amostra, o código de unidade primária de amostragem (setor) e o peso calibrado.

Os dados foram analisados de forma descritiva e exploratória para avaliar a distribuição e caracterizar a população estudada. As variáveis quantitativas foram descritas por medidas de tendência central e de dispersão. As variáis qualitativas foram descritas em números absolutos e proporções. Para análise das diferenças de média foram empregados os testes estatísticos $t$ de Student e ANOVA, e para as diferenças entre as variáveis categóricas o teste de qui-quadrado de Pearson. A descrição da população do estudo foi realizada segundo sexo e presença ou ausência de doença renal crônica.

Também foi realizada a análise bivariada com objetivo de explorar a associação das diferentes variáveis e o objeto de estudo. Modelos de regressão logística estimaram a magnitude de associação, em odds ratio (OR), entre a variável dependente DRC e as variáveis independentes.

$\mathrm{Na}$ análise múltipla foram selecionadas para inclusão aquelas variáveis que apresentaram valor de $\mathrm{p}$ menor que 0,20 na análise bruta, sendo analisada a magnitude das variáveis ajustadas pelas demais variáveis significativas. O método backward foi utilizado na seleção das variáveis para a análise multivariada. Foi testado, ainda, um modelo em que foram mantidas as variáveis 
sexo, grupo etário e IMC. Na análise da síndrome metabólica não foram incluídas as variáveis HAS, DM e dislipidemia. Foram testadas interações da idade e do sexo com a DRC, não sendo observado modificação de efeito. O nível de significância considerado foi de $\alpha=0,05$.

Todas as análises levaram em conta o efeito do desenho amostral e os pesos calibrados das observações, sendo os resultados das observações demonstrados por ' $n$ ' e os resultados considerando os pesos calibrados para extrapolação para a população pelo ' $n$ expantido $(\mathrm{N})$ '. Para tanto foi utilizado o método de máxima pseudo-verossimilhança (MPV) considerando os pesos amostrais e as informações estruturais do plano amostral. Nesse método os pesos são incorporados à análise através das equações de estimação dos parâmetros e de estimação da matriz de covariância dos estimadores ${ }^{22}$. Sendo as inferências avaliadas pela estatística de Wald baseado no plano amostral juntamente com a distribuição $\mathrm{F}^{23}$.

O presente estudo atendeu aos dispositivos da Resolução CNS 466/2012 que trata da ética em pesquisas envolvendo seres humanos. Tendo sido aprovado pelo Comitê de Ética em Pesquisas (CEP) da Universidade Federal do Acre. Todos os participantes assinaram o Termo de Consentimento Livre e Esclarecido (TCLE).

\section{Resultados}

A prevalência de DRC foi de $6,2 \%$ segundo a fórmula CKD-EPI, sendo a prevalência de TFG $<60 \mathrm{ml} / \mathrm{min} / 1,73 \mathrm{~m}^{2}$ de $0,6 \%$, tendo $0,2 \%$ dos indivíduos no estágio 3 e $0,4 \%$ no estágio 5 . A prevalência entre os adultos de prognóstico de risco leve, moderado e alto foi de 5,2\%, 0,6\% e $0,5 \%$, respectivamente. Entre os indivíduos com albuminúria $\geq 30 \mathrm{mg} / \mathrm{g}$ a prevalência foi de $6,0 \%$ (Tabela 1). A prevalência de DRC segundo autorrelato foi de $0,7 \%$.

A presença de DRC foi maior entre as mulheres, também entre os sujeitos com 40 a 59 anos, de cor da pele não-branca, com menor escolaridade, com companheiro e entre os sedentários. Dos indivíduos com DRC, cerca de 5,0\% foram classificados como fumante ou ex-fumante e $3,4 \%$ relatou consumir bebida alcoólica (Tabela 2).

Dentre as morbidades avaliadas, os portadores de DRC tiveram a maior prevalência de diabetes, aproximadamente 20,0\%. Houve diferenças estatisticamente significativas na distribuição segundo a presença de DRC nas variáveis HAS, diabetes e internação nos últimos 12 meses, ficando boderline a presença de síndrome metabólica (Tabela 3).

Para avaliar a mudança da OR com a introdução de variáveis potencialmente confundidoras foram estimados modelos com aquelas variáveis com valor de $\mathrm{p}<0,20$ na análise bivariada, conservando quando valor de $\mathrm{p}<0,05$. Assim, observou-se associação estatisticamente significativa entre DRC e sexo feminino $(\mathrm{OR}=2,41)$, diabetes $(\mathrm{OR}=4,67)$ e HAS $(\mathrm{OR}=1,98)$. Levando-se em consideração a importância dada pela literatura ao papel do sexo e do IMC em relação à DRC, realizou-se ajuste mantendo essas variáveis. A OR de DM apresentou leve aumento da magnitude, enquanto a de HAS permaneceu constante (Tabela 4$)$.

\section{Discussão}

A prevalência de DRC nos adultos (18-59 anos) foi de $6,2 \%$ estando significativamente associada ao sexo e às morbidades HAS e diabetes. $\mathrm{Na} \mathrm{Co-}$ reia, a prevalência observada foi de $7,9 \%$, sendo de $2,2 \%$ ao se considerar TFG $<60 \mathrm{ml} / \mathrm{min} / 1,73$ $\mathrm{m}^{2}$ e de $6,5 \%$ para albuminúria, dados referente ao inquérito nacional realizado entre 2011 e 2012, com indivíduos $\geq 19$ anos $^{24}$, valores estes maiores dos que os observados no presente estudo.

A prevalência de albuminúria geralmente é maior que a de TFG $<60 \mathrm{ml} / \mathrm{min} / 1,73 \mathrm{~m}^{2}$, pois a proteinúria está presente antes do comprometimento da função renal ${ }^{25}$. Além disso, a albuminúria é igualmente importante para o prognóstico das doenças cardiovasculares (DCV) e de mortalidade ${ }^{26}$. Os indivíduos nos estágios iniciais da DRC possuem maior risco de desenvolver DCV e aqueles que já as possuem tendem ao seu agravamento $^{27}$. As DCV e a DRC estão inter-relacionadas, uma vez que a doença de um órgão causa disfunção no outro, chegando em situações graves a falha de ambos ${ }^{28}$.

Os estudos sobre prevalência de DRC utilizando os critérios KDIGO (2012) ainda são escassos no Brasil. A maioria dos estudos publicados utilizam a TFG verificada por diferentes fórmulas e biomarcadores ou em grupos específicos. Em estudo transversal realizado com adultos atendidos pela Estratégia de Saúde da Família (ESF) em Goiânia, Goiás, a TFG, segundo a fórmula CG, < $60 \mathrm{ml} / \mathrm{min} / 1,73 \mathrm{~m}^{2}$ foi de 6,7 e a albuminúria $\geq$ $30 \mathrm{mg} / \mathrm{g}$ foi de $21,2 \%{ }^{29}$. Em estudo realizado em Tubarão, Santa Catarina, com indivíduos de 18 a 59 anos, a prevalência de TFG, segundo a fór- 
Tabela 1. Prevalência por categorias de risco do prognóstico de evolução da DRC mediante TFG (fórmula CKD-EPI) e albuminúria em adultos de Rio Branco, Acre, 2014.

\begin{tabular}{|c|c|c|c|c|c|c|c|c|c|c|c|}
\hline \multirow{3}{*}{\multicolumn{2}{|c|}{$\begin{array}{l}\text { Categorias de } \\
\text { risco da DRC }\end{array}$}} & \multirow{2}{*}{\multicolumn{2}{|c|}{ Total }} & \multicolumn{6}{|c|}{ Albuminúria } & \multirow{2}{*}{\multicolumn{2}{|c|}{$\begin{array}{c}\text { DRC } \\
\left(\mathrm{TFG}^{*} \mathrm{e} / \mathrm{ou}\right. \\
\text { Albuminúria })\end{array}$}} \\
\hline & & & & \multicolumn{2}{|r|}{ A1 $(<30)$} & \multicolumn{2}{|c|}{ A2 (30-299) } & \multicolumn{2}{|c|}{ A3 $(\geq 300)$} & & \\
\hline & & $\mathbf{n}$ & $N \exp (\%)$ & $\mathbf{n}$ & $N \exp (\%)$ & $\mathbf{n}$ & $N \exp (\%)$ & $\mathbf{n}$ & $N \exp (\%)$ & $\mathbf{n}$ & $\mathrm{N} \exp (\%)$ \\
\hline 1 & $\geq 90$ & 570 & $193.201(91,2)$ & 520 & $178.652(86,1)$ & 31 & $9.142(4,4)$ & 04 & $1.038(0,5)$ & 35 & $10.180(4,8)$ \\
\hline 2 & $60-89$ & 74 & $17.469(8,2)$ & 66 & $15.781(7,6)$ & 07 & $1.449(0,7)$ & 01 & $238(0,1)$ & 08 & $1.688(0,8)$ \\
\hline $3 a$ & $45-59$ & 02 & $246(0,1)$ & 02 & $246(0,1)$ & - & - & - & & 02 & $246(0,1)$ \\
\hline $3 b$ & $30-44$ & 01 & $123(0,1)$ & - & - & 01 & $123(0,1)$ & - & & 01 & $123(0,1)$ \\
\hline 4 & $15-29$ & - & - & - & - & - & - & - & - & - & - \\
\hline 5 & $<15$ & 02 & $863(0,4)$ & 01 & $310(0,2)$ & 01 & $553(0,2)$ & - & - & 02 & $863(0,4)$ \\
\hline Total & & 649 & $211.902(100,0)$ & 589 & $194.989(94,0)$ & 40 & $11.267(5,4)$ & 05 & $1.276(0,6)$ & 48 & $13.100(6,2)$ \\
\hline & & & & derac & Alto & & & & & & \\
\hline
\end{tabular}

Tabela 2. Prevalência de DRC segundo características sociodemográficas e de hábitos de vida dos adultos em Rio Branco, Acre, 2014.

\begin{tabular}{|c|c|c|c|c|c|c|c|c|c|c|}
\hline \multirow{3}{*}{ Variáveis } & \multirow{2}{*}{\multicolumn{2}{|c|}{ Total }} & \multicolumn{6}{|c|}{ DRC } & \multirow{3}{*}{$\mathrm{OR}_{\text {Bruta }}(\mathrm{IC} 95 \%)$} & \multirow{3}{*}{$\begin{array}{l}\text { p- } \\
\text { valor }\end{array}$} \\
\hline & & & \multicolumn{3}{|c|}{ Sim } & \multicolumn{3}{|c|}{ Não } & & \\
\hline & $\mathbf{n}$ & $N \exp$ & $\mathbf{n}$ & $\mathrm{N} \exp$ & $\%$ & $\mathbf{n}$ & $\mathrm{N} \exp$ & $\%$ & & \\
\hline Sexo & & & & & & & & & & 0,048 \\
\hline Masculino & 197 & 101.399 & 11 & 4.099 & 4,0 & 186 & 97.300 & 96,0 & 1 & \\
\hline Feminino & 452 & 110.503 & 37 & 9.001 & 8,1 & 415 & 101.502 & 91,9 & $2,11(1,01-4,41)$ & \\
\hline Grupo etário (anos) & & & & & & & & & & 0,271 \\
\hline $18-39$ & 348 & 146.447 & 23 & 7.873 & 5,4 & 325 & 138.574 & 94,6 & 1 & \\
\hline $40-59$ & 301 & 65.455 & 25 & 5.227 & 8,0 & 276 & 60.228 & 92,0 & $1,53(0,71-3,29)$ & \\
\hline Cor da pele & & & & & & & & & & 0,537 \\
\hline Branca & 120 & 38.427 & 08 & 1.948 & 5,1 & 112 & 36.478 & 94,9 & 1 & \\
\hline Não branca & 529 & 173.475 & 40 & 11.152 & 6,4 & 489 & 162.324 & 93,6 & $1,29(0,57-2,91)$ & \\
\hline Escolaridade $^{\star}$ & & & & & & & & & & 0,645 \\
\hline $\begin{array}{l}\text { Ensino médio e } \\
\text { superior }\end{array}$ & 273 & 95.008 & 17 & 5.438 & 5,7 & 256 & 89.570 & 94,3 & 1 & \\
\hline $\begin{array}{l}\text { Até o ensino } \\
\text { fundamental }\end{array}$ & 362 & 112.943 & 30 & 7.451 & 6,6 & 332 & 105.492 & 93,4 & $1,16(0,60-2,25)$ & \\
\hline Situação conjugal $^{*}$ & & & & & & & & & & 0,683 \\
\hline $\begin{array}{l}\text { Com } \\
\text { companheiro }\end{array}$ & 304 & 96.193 & 23 & 6.471 & 6,7 & 281 & 89.722 & 93,3 & 1 & \\
\hline Sem companheiro & 342 & 114.823 & 25 & 6.629 & 5,8 & 317 & 108.194 & 94,2 & $0,85(0,38-1,89)$ & \\
\hline Atividade física ${ }^{\star}$ & & & & & & & & & & 0,420 \\
\hline Sim & 168 & 70.118 & 09 & 3.436 & 4,9 & 159 & 66.682 & 95,1 & 1 & \\
\hline Não & 478 & 140.532 & 39 & 9.664 & 6,9 & 439 & 130.868 & 93,1 & $1,43(0,59-3,50)$ & \\
\hline Tabagismo* & & & & & & & & & & 0,059 \\
\hline Não fumante & 353 & 122.179 & 32 & 9.280 & 7,6 & 321 & 112.899 & 92,4 & 1 & \\
\hline $\begin{array}{l}\text { Fumante e ex- } \\
\text { fumante }\end{array}$ & 291 & 88.348 & 15 & 3.524 & 4,0 & 276 & 84.824 & 96,0 & $0,50(0,25-1,03)$ & \\
\hline $\begin{array}{l}\text { Consumo de bebida } \\
\text { alcoólica }^{*}\end{array}$ & & & & & & & & & & 0,009 \\
\hline Não & 467 & 142.632 & 41 & 11.192 & 7,8 & 426 & 131.440 & 92,2 & 1 & \\
\hline Sim & 158 & 61.110 & 05 & 1.302 & 2,1 & 153 & 59.808 & 97,9 & $0,26(0,09-0,69)$ & \\
\hline
\end{tabular}

$\mathrm{N} \exp =\mathrm{N}$ expandido a partir dos pesos e o delineamento amostral; \% = proporção a partir do N exp.; p-valor = teste Quiquadrado de Pearson. *As diferenças em relação ao total são decorrentes de falta de informação na variável. 
Tabela 3. Prevalência de DRC segundo condições de saúde dos adultos em Rio Branco, Acre, 2014.

\begin{tabular}{|c|c|c|c|c|c|c|c|c|c|c|}
\hline \multirow{3}{*}{ Variáveis } & \multirow{2}{*}{\multicolumn{2}{|c|}{ Total }} & \multicolumn{6}{|c|}{ DRC } & \multirow{3}{*}{ OR $_{\text {Bruta }}$ IC95\% } & \multirow{3}{*}{$\begin{array}{c}\text { p- } \\
\text { valor }\end{array}$} \\
\hline & & & \multicolumn{3}{|c|}{ Sim } & \multicolumn{3}{|c|}{ Não } & & \\
\hline & $\mathbf{n}$ & $N \exp$ & $\mathbf{n}$ & $N \exp$ & $\%$ & $\mathbf{n}$ & $N \exp$ & $\%$ & & \\
\hline Autoavaliação de saúde ${ }^{\star}$ & & & & & & & & & & 0,468 \\
\hline Muito boa, boa e regular & 557 & 186.385 & 39 & 11.168 & 6,0 & 518 & 175.217 & 94,0 & 1 & \\
\hline Ruim, muito ruim & 89 & 24.201 & 09 & 1.931 & 8,0 & 80 & 22.270 & 92,0 & $1,36(0,58-3,18)$ & \\
\hline $\operatorname{IMC}\left(\mathrm{m} / \mathrm{kg}^{2}\right)^{*}$ & & & & & & & & & & 0,525 \\
\hline$<25,0$ & 254 & 90.560 & 17 & 5.332 & 5,9 & 237 & 85.228 & 94,1 & 1 & \\
\hline $25,0-29,9$ & 240 & 75.998 & 14 & 4.086 & 5,4 & 226 & 71.912 & 94,6 & $0,91(0,39-2,12)$ & \\
\hline$\geq 30,0$ & 139 & 38.699 & 15 & 3.245 & 8,4 & 124 & 35.454 & 91,6 & $1,46(0,50-4,24)$ & \\
\hline Hipertensão* & & & & & & & & & & 0,018 \\
\hline Não & 474 & 166.004 & 27 & 8.584 & 5,2 & 447 & 157.420 & 94,8 & 1 & \\
\hline Sim & 162 & 40.633 & 21 & 4.515 & 11,1 & 141 & 36.118 & 88,9 & $2,29(1,16-4,52)$ & \\
\hline Diabetes & & & & & & & & & & 0,024 \\
\hline Não & 617 & 201.269 & 40 & 10.994 & 5,5 & 577 & 190.275 & 94,5 & 1 & \\
\hline Sim & 32 & 10.633 & 08 & 2.105 & 19,8 & 24 & 8.528 & 80,2 & $4,27(1,23-14,88)$ & \\
\hline Dislipidemia & & & & & & & & & & 0,335 \\
\hline Não & 158 & 59.596 & 10 & 2.918 & 4,9 & 148 & 56.678 & 95,1 & 1 & \\
\hline Sim & 491 & 152.306 & 38 & 10.181 & 6,7 & 453 & 142.125 & 93,3 & $1,39(0,70-2,76)$ & \\
\hline Síndrome metabólica ${ }^{\star}$ & & & & & & & & & & 0,050 \\
\hline Não & 537 & 181.889 & 34 & 10.057 & 5,5 & 503 & 171.832 & 94,5 & 1 & \\
\hline Sim & 105 & 27.211 & 14 & 3.043 & 11,2 & 91 & 24.168 & 88,8 & $2,15(1,00-4,62)$ & \\
\hline $\begin{array}{l}\text { Outras DCV autorreferidas } \\
\text { (ICC, IAM, AVE, } \\
\text { Arritmia) }{ }^{*}\end{array}$ & & & & & & & & & & 0,189 \\
\hline Não & 607 & 199.790 & 45 & 12.635 & 6,3 & 562 & 187.155 & 93,7 & 1 & \\
\hline Sim & 36 & 10.246 & 02 & 246 & 2,4 & 34 & 10.000 & 97,6 & $2,74(0,59-12,63)$ & \\
\hline Uso de medicamentos & & & & & & & & & & 0,162 \\
\hline Não & 405 & 145.751 & 24 & 7.775 & 5,3 & 381 & 137.976 & 94,7 & 1 & \\
\hline Sim & 244 & 66.151 & 24 & 5.325 & 8,0 & 220 & 60.826 & 92,0 & $1,55(0,83-2,90)$ & \\
\hline $\begin{array}{l}\text { Medicamentos de uso } \\
\text { contínuo* }^{*}\end{array}$ & & & & & & & & & & 0,718 \\
\hline Não & 468 & 154.912 & 32 & 9.289 & 6,0 & 436 & 145.624 & 94,0 & 1 & \\
\hline Sim & 148 & 47.297 & 12 & 2.642 & 5,3 & 148 & 47.297 & 94,7 & $0,88(0,42-1,83)$ & \\
\hline $\begin{array}{l}\text { Internação nos últimos } 12 \\
\text { meses }^{\star}\end{array}$ & & & & & & & & & & 0,016 \\
\hline Não & 533 & 177.058 & 33 & 8.945 & 5,1 & 500 & 168.113 & 94,9 & 1 & \\
\hline Sim & 91 & 24.655 & 12 & 3.139 & 12,7 & 79 & 21.516 & 87,3 & $2,74(1,22-6,15)$ & \\
\hline
\end{tabular}

$\mathrm{N}$ exp = N expandido a partir dos pesos e o delineamento amostral; \% = proporção a partir do N.; p-valor = teste Qui-quadrado de Pearson. *As diferenças em relação ao total são decorrentes de falta de informação na variável.

mula CKD-EPI, $<60 \mathrm{ml} / \mathrm{min} / 1,73 \mathrm{~m}^{2}$ foi $1,4 \%{ }^{30}$, maior que a encontrada em Rio Branco.

A prevalência de DRC tende a ser maior nas mulheres, conforme observado em estudo realizado nos EUA, em que a doença nos estágios 1 a 4 foi de 15,9\% nas mulheres e de $13,5 \%$ nos homens, no período de 1999 a $2012^{31}$. No entanto, nos homens a doença tende a progredir mais rapidamente para a forma mais grave, o que explica a sua maior frequência nas terapias renais subs- titutivas $^{32,33}$. Essa diferença está relacionada aos hormônios sexuais, onde os femininos tendem a retardar a progressão da $\mathrm{DRC}^{34}$, bem como à estrutura renal, à hemodinâmica sistêmica e renal, ao metabolismo lipídico e à pressão $\operatorname{arterial}^{35}$. No presente estudo houve maior prevalência de DRC entre as mulheres.

A diferença da prevalência existente entre o autorrelato e a avaliação segundo os critérios da KDIGO (2012) pode ocorrer em decorrência dos 
Tabela 4. Descrição dos fatores associados à DRC em adultos de Rio Branco, Acre, 2014.

\begin{tabular}{|c|c|c|}
\hline Variáveis & $\begin{array}{c}\text { OR (IC 95\%) } \\
\text { Modelo } 1\end{array}$ & $\begin{array}{c}\text { OR (IC 95\%) } \\
\text { Modelo } 2\end{array}$ \\
\hline \multicolumn{3}{|l|}{ Sexo } \\
\hline Masculino & 1 & 1 \\
\hline Feminino & $2,60(1,21-5,57)$ & $2,41(1,14-5,12)$ \\
\hline \multicolumn{3}{|c|}{ Grupo etário (anos) } \\
\hline $18-39$ & - & 1 \\
\hline 40-59 & - & $0,99(0,49-2,01)$ \\
\hline \multicolumn{3}{|l|}{$\operatorname{IMC}\left(\mathrm{Kg} / \mathrm{m}^{2}\right)$} \\
\hline$<25,0$ & - & 1 \\
\hline $25,0-29,9$ & - & $0,74(0,32-1,73)$ \\
\hline$\geq 30,0$ & - & $0,99(0,33-3,04)$ \\
\hline \multicolumn{3}{|l|}{ Diabetes } \\
\hline Não & 1 & 1 \\
\hline Sim & $4,32(1,25-14,94)$ & $4,67(1,28-17,03)$ \\
\hline \multicolumn{3}{|l|}{ Hipertensão } \\
\hline Não & 1 & 1 \\
\hline Sim & $1,98(1,05-3,73)$ & $1,98(1,16-3,37)$ \\
\hline
\end{tabular}

indivíduos terem conhecimento da DRC - ou se consideraram portadores - quando submetidos à terapia renal substitutiva. Esse fato é um alerta para a ocorrência de encaminhamentos tardios para acompanhamento de pacientes portadores de DRC, o que resulta em aumento da mortalidade $^{36}$.

Em Taiwan, na China, a prevalência de autorrelato de DRC de 1,4\% e, segundo TFG e albuminúria, foi de $9,5 \%{ }^{37}$. Em estudo de base populacional, realizado com 60.202 indivíduos com idade $\geq 18$ anos, o autorrelato de DRC resultou em uma prevalência de $1,4 \%$ na população brasileira $^{32}$.

No presente estudo, a DRC na população esteve associada à HAS. A HAS e a DRC possuem uma relação de causa e efeito bilateral, ou seja, tanto a DRC leva à hipertensão secundária, como a hipertensão, de qualquer etiologia, pode levar à insuficiência renal com aumento da PA acompanhada de proteinúria ${ }^{38}$. Nos EUA, a prevalência de HAS entre portadores de DRC alcança 86\%, enquanto que na população geral sua prevalência é de $29 \%{ }^{39,40}$. Os indivíduos com PA de aproximadamente $180 / 100 \mathrm{mmHg}$ têm 15 vezes maior chance de desenvolver DRC que os indivíduos com PA normal ${ }^{41}$. A fisiopatologia da DRC associada à HAS apresenta diferentes mecanismos, entre eles a desregulação de sódio, o aumento de atividade do sistema renina-angiotensina-aldosterona e a disfunção endotelial ${ }^{38}$.

Em estudo transversal realizado nas ilhas caribenhas de Saint Kitts e Nevis, envolvendo 1.978 indivíduos com 18 anos e mais, os portadores de DRC tiveram 2,98 vezes maior chance de ter HAS quando comparados aos sem DRC ${ }^{21}$. Também foi observada associação da HAS com a DRC, em outros estudos de base populacional, como os da população rural no sul da Índia (OR: 2,2; IC95\%: 1,46-3,36) $)^{42}$, e em León, Nicarágua (OR: 2,07; IC95\%: 1,43-3,02) ${ }^{43}$.

$\mathrm{O}$ diabetes também esteve associado à presença de DRC segundo a TFG e albuminúria em adultos. Outros trabalhos reportaram tal associação $^{21,44}$. O mecanismo de lesão renal no indivíduo diabético inclui a alteração na perfusão, o aumento da pressão e a hiperfiltração glomerular que leva à proteinúria e resulta em glomeruloesclerose com diminuição da $\mathrm{TFG}^{45}$. A presença de HAS e condições genéticas podem alterar esse processo, tendo ou não albuminúria e presença de lesão característica da doença renal ${ }^{46,47}$. No Brasil, indivíduos com proteinúria eram na maioria diabéticos ou hipertensos ${ }^{48}$.

Entre os diabéticos, o aumento da proteinúria está associado ao elevado risco de $\mathrm{DCV}^{49}$. A ocorrência de DCV reduz a expectativa de vida neste grupo de pacientes. Estudos têm demonstrado um crescimento da mortalidade entre os pacientes com diabetes e DRC, em comparação com aqueles com DRC mas sem diabetes ${ }^{50,51}$.

A detecção precoce da DRC na população geral por meio da proteinúria é reconhecido como um método prático, de baixo custo e eficaz para ser realizado no $\mathrm{Brasil}^{48}$. A detecção precoce voltada a grupos de risco, como hipertensos e diabéticos é defendida ${ }^{52}$. No entanto, no Brasil a adoção de métodos para detecção somente em grupos específicos parece não ser a melhor opção, uma vez que, a DRC esteve fortemente associada às condições de desigualdade social como baixa escolaridade e cor da pele negra ou parda e em indígenas ${ }^{20}$. Além disso, entre os diabéticos do tipo 2, a avaliação somente pela albuminúria pode resultar em perda substancial de detecção de caso de DRC sem proteinúria ${ }^{53}$.

Destarte há de se reconhecer algumas limitações na presente pesquisa, como o desenho transversal do estudo, que impede a realização de inferências causais. Entretanto, esse tipo de estudo tem relevância na análise exploratória dos fatores, além de ser uma ferramenta indispensável na detecção precoce da DRC na população geral. 
A inferência para a população dos resultados encontrados é um ponto salutar do presente estudo, uma vez que foi considerado nas análises o efeito do plano amostral incorporando os pesos das observações, o estrato e a unidade de amostragem primária.

Outro ponto a ser considerado é o diagnóstico pontual da DRC baseado em uma amostra de sangue e urina empregados no cálculo da TFG e da albuminúria, visto que a confirmação da DRC dá-se pela persistência da redução da TFG e/ou aumento da albuminúria. No entanto, para detecção em estudos de base populacional esse é um método possível de ser empregado que vem sendo utilizado em diversos outros estudos ${ }^{20,21,24}$. Como medida de padronização dos procedimentos de análise e garantia da qualidade dos resultados, todos os exames laboratoriais foram realizados num mesmo laboratório. Também a TFG foi calculada por meio da creatina sérica usando a equação CKD-EPI o que reduz a possibilidade de erro na estimativa da $\mathrm{TFG}^{54}$.

Ressalta-se, ainda, a avaliação dos parâmetros clínicos para definição da DRC e das morbidades utilizadas nas variáveis independentes, destacando o caráter inédito e rigoroso utilizado nas análises das informações aqui apresentadas. Também cabe destacar que a presente pesquisa trouxe a elucidação informações representativa de adultos sobre o tema DRC em Rio Branco, Acre, apresentando os resultados dos estágios da DRC na população, baseado na definição proposta pelo KDIGO (2012). Tal fato possibilita comparações com a literatura internacional e nacional, sendo que na maioria dos estudos brasileiros este procedimento foi realizado com populações específicas ou baseados em autorrelato da DRC, ou ainda, realizados com indivíduos em diálise ou baseados na TFG ou proteinúria, o que limita os achados.

\section{Conclusão}

A prevalência de DRC foi alta entre os adultos de 18 a 59 anos. Os fatores associados à DRC, após ajuste, em adultos foram sexo, HAS e diabetes. O presente estudo é um primeiro e importante esforço para a detecção da DRC e dos fatores a ela associados na população de Rio Branco, Acre, ressaltando-se a importância do reconhecimento precoce da DRC para minimizar e prevenir do agravamento da doença para estágios mais avançados.

Somente conhecer os fatores relacionados à DRC não é o suficiente, pois é crescente o número de indivíduos com essa patologia, sendo urgente estratégias de saúde voltadas ao acompanhamento da função renal. Essas devem contemplar tanto a população geral que, cada vez mais, está envelhecendo e sofrendo, como grupos populacionais mais suscetíveis, como diabéticos e hipertensos, para o tratamento adequado e detecção precoce da DRC. Uma estratégia em saúde pública para reduzir os casos de DRC em mais da metade seria o acompanhamento para detecção precoce, com base em marcadores como proteinúria no programa Hiperdia.

\section{Financiamento}

Os recursos financeiros para realização da pesquisa foram obtidos junto ao Conselho Nacional de Desenvolvimento Científico e Tecnológico $(\mathrm{CNPq})$, para apoio a pesquisas estratégicas para o Sistema de Saúde pela Rede Brasileira de Avaliação de Tecnologias em Saúde (REBRATS) e, também, junto à Fundação de Amparo à Pesquisa do Acre (FAPAC), do Programa de Pesquisa para o SUS: gestão compartilhada em saúde (MS/ CNPq/FAPAC/SESACRE). 


\section{Agradecimentos}

À Profa. Dra. Margareth Crisóstomo Portela por suas contribuições nas análises e interpretações dos dados, ao Prof. Dr. Sérgio Koifman (in Memoriam) pela idealização e incentivo para a realização da presente pesquisa e à Secretaria Municipal de Saúde de Rio Branco pelo acolhimento e atendimento de todos os indivíduos identificados com alterações de saúde durante a pesquisa.

\section{Colaboradores}

TLM Amaral e CA Amaral: concepção do projeto, análise e interpretação dos dados; redação do artigo; aprovação final da versão a ser publicada; responsáveis por todos os aspectos do trabalho na garantia da exatidão e integridade de qualquer parte da obra. MTL Vasconcellos e GTR Monteiro: análise e interpretação dos dados; revisão crítica relevante do conteúdo intelectual; aprovação final da versão a ser publicada; responsáveis por todos os aspectos do trabalho na garantia da exatidão e integridade de qualquer parte da obra.

\section{Referências}

1. Bastos MG, Kirsztajn GM. Chronic kidney disease: importance of early diagnosis, immediate referral and structured interdisciplinary approach to improve outcomes in patients not yet on dialysis. J Bras Nefrol 2011; 33(1):93-108.

2. Cusumano AM, Bedat MCG. Chronic Kidney Disease in Latin America: Time to Improve Screening and Detection. Clin J Am Soc Nephrol 2008; 3(2):594-600.

3. Organização Mundial da Saúde (OMS). Prevenção de Doenças Crônicas um investimento vital [Internet]. Geneva: OMS; 2005 [acessado 2017 Jan 27]. Disponível em: http://www.who.int/chp/chronic_disease_report/part1_port.pdf

4. Eckardt K, Bansal N, Coresh J, Evans M, Grams ME, Herzog CA, James MT, Heerspink HJL, Pollock CA, Stevens PE, Tamura MK, Tonelli MA, Wheeler DC, Winkelmayer WC, Cheung M, Hemmelgarn BR, Conference Participants. Improving the prognosis of patients with severely decreased glomerular filtration rate (CKD G4+): conclusions from a Kidney Disease: Improving Global Outcomes (KDIGO) Controversies Conference. Kidney Int 2018; 93(6):1281-1292.

5. Brasil. Ministério da Saúde (MS). Portaria no 1.168, de 15 de junho de 2004. Institui a Política Nacional de Atenção ao Portador de Doença Renal, a ser implantada em todas as unidades federadas, respeitadas as competências das três esferas de gestão. Diário Oficial da União; 2004.

6. Kirsztajn GM, Salgado Filho N, Draibe SA, Pádua Netto MV, Thome FS, Souza E, Bastos MG. Fast Reading of the KDIGO 2012: Guidelines for evaluation and management of chronic kidney disease in clinical practice. J Bras Nefrol 2014; 36(1):63-73.

7. Levey AS, Eckardt K-U, Tsukamoto Y, Levin A, Coresh J, Rossert J, Zeeuw DD, Hostetter TH, Lameire N, Eknoyan G. Definition and classification of chronic kidney disease: a position statement from Kidney Disease: Improving Global Outcomes (KDIGO) Kidney Int 2005; 67(6):2089-2100.

8. Cueto-Manzano AM, Cortés-Sanabria L, MartínezRamírez HR, Rojas-Campos E, Gómez-Navarro B, Castillero-Manzano M. Prevalence of chronic kidney disease in an adult population. Arch Med Res 2014; 45(6):507-513.

9. Cochran WG. Sampling techniques. $33^{\mathrm{a}}$ ed. New York: John Wiley \& Sons; 1977.

10. Silva PLN. Calibration estimation: when and why, how much and how [Internet]. Rio de Janeiro: IBGE; 2004 [acessado 2017 Jan 29]. Disponível em: http://biblioteca.ibge.gov.br/biblioteca-catalogo?id=281040\&view $=$ detalhes

11. Madeira JL, Simões CCS. Estimativas preliminares da população urbana e rural segundo as unidades da federação, de 1960/1980 por uma nova metodologia. Rev Bras Estat 1972; 33:3-11.

12. Amaral TLM, Amaral CA, Portela MC, Monteiro GTR, Vasconcellos MTL. Study of Chronic Diseases (Edoc): methodological aspects. Rev Saúde Pública 2019; 53:8.

13. American College of Sports Medicine. Manual do ACSM para avaliação da aptidão física relacionada à saúde. Rio de Janeiro: Guanabara Koogan; 2006. 
14. World Health Organization (WHO). Obesity: preventing and managing the global epidemic [Internet]. Geneva: WHO; 2000 [acessado $2015 \mathrm{Abr}$ 8]. Disponível em: http://www.who.int/nutrition/publications/obesity/WHO_TRS_894/en/

15. Sociedade Brasileira de Cardiologia. Sociedade Brasileira de Hipertensão. Sociedade Brasileira de Nefrologia. VI Diretrizes brasileiras de hipertensão. Arq Bras Cardiol 2010; 95(S1):1-51.

16. Improving Global Outcomes (KDIGO) CKD Work Group. KDIGO 2012 Clinical Practice Guideline for the Evaluation and Management of Chronic Kidney Disease. Kidney Int Suppl 2013; 3(Supl. 1):1-150.

17. Sociedade Brasileira de Diabetes. Diretrizes da Sociedade Brasileira de Diabetes: 2013-2014 [Internet]. In: Oliveira JEP, Vencio S, organizadores. São Paulo: AC Farmacêutica; 2014. Disponível em: http://www.diabetes.org.br/images/pdf/diretrizes-sbd.pdf

18. Xavier HT, Izar MC, Faria Neto JR, Assad MH, Rocha VZ, Sposito AC, Fonseca FA, Snatos JE, Santos RD, Bertolami MC, Faludi AA, Martinez TLR, Diament J, Guimarães A, Forti NA, Moriguchi E, Chagas ACP, Coelho OR, Ramires JAF. V Diretriz Brasileira de Dislipidemias e Prevenção da Aterosclerose. Arq Bras Cardiol 2013; 101(4):1-20.

19. Sociedade Brasileira de Hipertensão. Sociedade Brasileira de Cardiologia. Sociedade Brasileira de Endocrinologia e Metabologia. Sociedade Brasileira de Diabetes. Associação Brasileira para Estudos da Obesidade. I Diretriz Brasileira de Diagnóstico e Tratamento da Síndrome Metabólica. Arq Bras Cardiol 2005; 84(Supl.1):3-28.

20. Barreto SM, Ladeira RM, Duncan BB, Schmidt MI, Lopes AA, Benseñor IM, Chor D, Griep RH, Vidigal PG, Ribeiro AI, Lotufo PA, Mill JG. Chronic kidney disease among adult participants of the ELSA-Brasil cohort: association with race and socioeconomic position. I Epidemiol Community Health 2016; 70(4):380-389.

21. Crews DC, Campbell KN, Liu Y, Bussue O, Nova, Young BA. Chronic kidney disease and risk factor prevalence in Saint Kitts and Nevis: a cross-sectional study. BMC Nephrol 2017; 18(1):7.

22. Pessoa DGCO, Silva PLN. Análise de dados amostrais complexos. São Paulo: Associação Brasileira de Estatística; 1998

23. Korn EL, Graubard BI. Simultaneous Testing of Regression Coefficients with Complex Survey Data: Use of Bonferroni t Statistics. Am Stat 1990; 44(4):270-276.

24. Ji E, Kim YS. Prevalence of chronic kidney disease defined by using CKD-EPI equation and albumin-tocreatinine ratio in the Korean adult population. Korean J Intern Med 2016; 31(6):1120-1130.

25. Mendes RS, Bregman R. Avaliação e metas do tratamento da proteinúria. Rev Bras Hipertens 2010; 17(3):174-177.

26. Ekart R, Bevc S, Hojs N, Knehtl M, Dvoršak B, Hojs R. Albuminuria is Associated with Subendocardial Viability Ratio in Chronic Kidney Disease Patients. Kidney Blood Press Res 2015; 40(6):565-574.
27. Kim H, Yoo TH, Choi KH, Oh KH, Lee J, Kim SW, Kim TH, Sung S, Han SH, KNOW-CKD Group. Baseline Cardiovascular Characteristics of Adult Patients with Chronic Kidney Disease from the KoreaN Cohort Study for Outcomes in Patients with Chronic Kidney Disease (KNOW-CKD). J Korean Med Sci 2017; 32(2):231-239.

28. Liu Y, Li S, Zeng Z, Wang J, Xie L, Li T, He Y, Qin X, Zhao J. Kidney stones and cardiovascular risk: a metaanalysis of cohort studies. Am J Kidney Dis 2014; 64(3):402-410.

29. Pereira ERS, Pereira AC, Andrade GB, Naghettini AV, Pinto FKMS, Batista SR, Marques SM. Prevalence of chronic renal disease in adults attended by the family health strategy. J Bras Nefrol 2016; 38(1):22-30.

30. Schaefer JCF, Pereira MS, Jesus CR, Schuelter-Trevisol F, Trevisol DJ. Kidney function estimate among subjects aged 18-59 years in Tubarão, Santa Catarina: A population-based study. J Bras Nefrol 2015; 37(2):185-191

31. Centers for Disease Control and Prevention (CDC). Chronic Kidney Disease Surveillance System-United States [Internet]. Whashington: CDC; 2017 [acessado 2017 Jan 27]. Disponível em: https://nccd.cdc.gov/ $\mathrm{CKD} /$ detail.aspx?Qnum $=\mathrm{Q} 10$

32. Moura L, Andrade SSCA, Malta DC, Pereira CA, Passos JEF. Prevalence of self-reported chronic kidney disease in Brazil: National Health Survey of 2013. Rev Bras Epidemiol 2015; 18:181-191.

33. Neugarten J, Golestaneh L. Gender and the prevalence and progression of renal disease. Adv Chronic Kidney Dis 2013; 20(5):390-395.

34. Kummer S, von Gersdorff G, Kemper MJ, Oh J. The influence of gender and sexual hormones on incidence and outcome of chronic kidney disease. $P e$ diatr Nephrol Berl Ger 2012; 27(8):1213-1219.

35. Chang P-Y, Chien L-N, Lin Y-F, Wu M-S, Chiu W-T, Chiou H-Y. Risk factors of gender for renal progression in patients with early chronic kidney disease. Medicine (Baltimore) 2016; 95(30):e4203.

36. Diegoli H, Silva MCG, Machado DSB, Cruz CERN. Encaminhamento tardio ao nefrologista e a associação com mortalidade em pacientes em hemodiálise. $J$ Bras Nefrol 2015; 37(1):32-37.

37. Xue L, Lou Y, Feng X, Wang C, Ran Z, Zhang X. Prevalence of chronic kidney disease and associated factors among the Chinese population in Taian, China. BMC Nephrol 2014; 15(1):205.

38. Hamrahian SM, Falkner B. Hypertension in Chronic Kidney Disease. Adv Exp Med Biol 2017; 956:307-325.

39. Egan BM, Zhao Y, Axon RN. US trends in prevalence, awareness, treatment, and control of hypertension, 1988-2008. JAMA 2010; 303(20):2043-2050.

40. Lash JP, Go AS, Appel LJ, He J, Ojo A, Rahman M, Townsend RR, Xie D, Cifelli D, Cohan J, Fink JC, Fischer MJ, Gadegbeku C, Hamm LL, Kusek JW, Landis JR, Narva A, Robinson N, Teal V, Feldman HI, Chronic Renal Insufficiency Cohort (CRIC) Study Group. Chronic Renal Insufficiency Cohort (CRIC) Study: baseline characteristics and associations with kidney function. Clin J Am Soc Nephrol CJASN 2009; 4(8):1302-1311. 
41. Tozawa M, Iseki K, Iseki C, Kinjo K, Ikemiya Y, Takishita S. Blood pressure predicts risk of developing end-stage renal disease in men and women. Hypertens Dallas Tex 1979 2003; 41(6):1341-1345.

42. Anupama YJ, Hegde SN, Uma G, Patil M. Hypertension is an important risk determinant for chronic kidney disease: results from a cross-sectional, observational study from a rural population in South India. J Hum Hypertens 2017; 31(5):327-332.

43. Lebov JF, Valladares E, Peña R, Peña EM, Sanoff SL, Cisneros EC, Colindres RE, Morgan DR, Hogan SL. A population-based study of prevalence and risk factors of chronic kidney disease in León, Nicaragua. Can J Kidney Health Dis 2015; 2:6.

44. Stojceva-Taneva O, Otovic NE, Taneva B. Prevalence of Diabetes Mellitus in Patients with Chronic Kidney Disease. Open Access Maced J Med Sci 2016; 4(1):7982.

45. Boer IH, Steffes MW. Glomerular filtration rate and albuminuria: twin manifestations of nephropathy in diabetes. J Am Soc Nephrol JASN 2007; 18(4):10361037.

46. Mrozikiewicz-Rakowska B, Maroszek P, Nehring P, Sobczyk-Kopciol A, Krzyzewska M, Kaszuba AM, Lukawska M, Chojnowska N, Kozka M, BujalskaZadrozny M, Ploski R, Krzymien J, Czupryniak L. Genetic and environmental predictors of chronic kidney disease in patients with type 2 diabetes and diabetic foot ulcer: a pilot study. J Physiol Pharmacol 2015; 66(5):751-761.

47. Schelling JR, Abboud HE, Nicholas SB, Pahl MV, Sedor JR, Adler SG, Arar NH, Bowden DW, Elston RC, Freedman BI, Goddard KAB, Guo X, Hanson RL, Ipp E, Iyengar SK, Jun G, Kao WHL, Kasinath BS, Kimmel PL, Klag MJ, Knowler WC, Nelson RG, Parekh RS, Quade SR, Rich SS, Saad MF, Scavini M, Smith MW, Taylor K, Winkler CA, Zager PG, Shah VO, Family Investigation of Nephropathy and Diabetes Research Group. Genome-wide scan for estimated glomerular filtration rate in multi-ethnic diabetic populations: the Family Investigation of Nephropathy and Diabetes (FIND). Diabetes 2008; 57(1):235-243.

48. Lima AO, Kesrouani S, Gomes RA, Cruz J, MastroianniKirsztajn G. Population screening for chronic kidney disease: a survey involving 38,721 Brazilians. Nephrol Dial Transplant 2012; 27(Supl. 3):iii135-138.
49. Solini A, Ferrannini E. Pathophysiology, prevention and management of chronic kidney disease in the hypertensive patient with diabetes mellitus. J Clin Hypertens Greenwich Conn 2011; 13(4):252-257.

50. Afkarian M, Sachs MC, Kestenbaum B, Hirsch IB, Tuttle KR, Himmelfarb J, de Boer IH. Kidney Disease and Increased Mortality Risk in Type 2 Diabetes. J Am Soc Nephrol 2013; 24(2):302-308.

51. Ang YG, Heng BH, Saxena N, Liew STA, Chong P-N. Annual all-cause mortality rate for patients with diabetic kidney disease in Singapore. J Clin Transl Endocrinol 2016; 4:1-6.

52. Hallan SI, Dahl K, Oien CM, Grootendorst DC, Aasberg A, Holmen J, Dekker FW. Screening strategies for chronic kidney disease in the general population: follow-up of cross sectional health survey. BMJ 2006; 333(7577):1047.

53. Kramer H, Molitch ME. Screening for kidney disease in adults with diabetes. Diabetes Care 2005; 28(7):1813-1816.

54. Park JI, Baek H, Jung HH. Prevalence of Chronic Kidney Disease in Korea: the Korean National Health and Nutritional Examination Survey 2011-2013. J Korean Med Sci 2016; 31(6):915-923.

Artigo apresentado em 17/02/2018

Aprovado em 24/04/2019

Versão final apresentada em 26/04/2019

Editores chefes: Romeu Gomes, Antônio Augusto Moura da Silva 